\title{
Globale Interaktion am Telefon? Die begrenzte Transnationalisierung von Callcenter- Dienstleistungen
}

\author{
Ursula Holtgrewe/Hannelore Mottweiler \\ Jessica Longen/Annika Schönauer
}

\begin{abstract}
Callcenter gelten mittlerweile vielfach als Paradebeispiele für die Möglichkeit, Arbeit technikunterstützt über Betriebe und Zeitzonen hinweg neu zu verteilen. Im englischsprachigen Raum - so der weitverbreitete Eindruck - werden diese Dienstleistungen ganz alltäglich ausgegliedert und verlagert. Gerade Indien wird häufig als Beleg für diese Praxis angeführt. Die technischen und praktischen Voraussetzungen sind offenkundig vorhanden; die Kostenvorteile scheinen eindeutig. Aber gibt es wirklich eine grenzenlos globalisierte Welt der Callcenter-Dienste? ${ }^{1}$
\end{abstract}

\section{Einführung}

Man hat Dienstleistungen im Kundenkontakt lange als weitgehend lokal gebunden betrachtet, weil hier, theoretisch betrachtet, Produktion und Konsum gleichzeitig stattfinden. Vernetzte Informations- und Kommunikationstechnik und immaterielle Produkte (wie Flugreservierungen oder Kreditkartenabrechnungen) ermöglichen es jedoch, Teile vieler Dienstleistungen räumlich getrennt zu erbringen. Das eröffnet Management und Unternehmen neue Optionen: Man kann die Kundendienste outsourcen, verkaufen, regional verlagern und sie insgesamt außerhalb von Branchengrenzen oder Tarifverträgen ansiedeln. Natürlich kann man die Arbeit auch ins Ausland („Offshoring“, oder „Nearshoring“ in benachbarte Länder) oder in Regionen mit niedrigeren Lohnkosten verschieben, indem man dort Tochterunternehmen gründet oder dortige Dienstleister beauftragt. Die bloße technisch-organisatorische Möglichkeit aber bestimmt noch nicht die reale Praxis von Betrieben und Managern. Aus verschiedenen Untersuchungen transnationaler Arbeitsteilungen (Djelic/Quack 2003; Flecker et al. 2008) wird deutlich, dass Unternehmensstrategien und Restrukturierungsentscheidungen weiterhin auch vom institutionellen Rahmen, von regionalen und nationalen Pfadabhängigkeiten und von kulturellen Mustern geprägt sind. Dies gilt auch für Callcenter (Batt et al. 2009a; Holtgrewe 2009; Sørensen/Weinkopf 2009).

In diesem Beitrag untersuchen wir das Ausmaß an „realer“ Transnationalisierung in Callcentern und das Profil der Callcenter, die grenzüberschreitend arbeiten. Die Daten aus dem Global Call Center Industry Project (GCC) ${ }^{2}$ zeigen, dass die Transnationalisierung begrenzt ist. Transnational arbeitende Callcenter konzentrieren sich unter den Outsourcing-Dienstleistern mit standardisierter und reglementierter Arbeit. Gleichwohl gibt es länderspezifisch unterschiedliche Muster. Das branchenweite und länderübergreifende Bild ergänzen wir um eine Einzelfallstudie des deutschsprachigen Kundendienstes eines US-basierten Elektronik-Multis. Dieser Fall zeigt, wie transnationale Strategien sich zwischen globalen Möglichkeiten und realer Praxis bewegen. Indem wir die internationale Befragung mit einer Fallstudie kombinieren, tragen wir zu einem mehrschichtigen Bild des Callcenter-Einsatzes bei, das die Entwicklung transnationaler Dienstleistungen weder aufgrund der Praxen von multinationalen (Pionier-)Unternehmen überschätzt, noch aufgrund des derzeitigen Verbreitungsgrads unterschätzt.

\footnotetext{
Der vorliegende Beitrag ist eine leicht gekürzte und aktualisierte Übersetzung des Aufsatzes "Global or embedded service work? The (limited) transnationalisation of the call centre industry" (Holtgrewe et al. 2009). Die zugrunde liegenden Forschungen wurden finanziert von der Hans-Böckler-Stiftung, dem Jubiläumsfonds der Österreichischen Nationalbank, dem österreichischen Fonds zur Förderung der wissenschaftlichen Forschung (FWF) und dem Graduiertenkolleg "Topologie der Technik" an der TU Darmstadt. Wir danken den Kollegen des Global Call Center Industry Project und besonders Rose Batt, David Holman, Ursula Huws, Rudi Schmiede und Karen Shire für Anregungen und Kommentare. Unser Dank gilt auch unseren anonymen Interviewpartnern.
}

2 Das Global Call Center Industry Project hat zwischen 2004 und 2006 eine standardisierte Managementbefragung in nahezu 2.500 Centern in 17 Ländern durchgeführt, die zusammengerechnet 475.000 Beschäftigte haben. Die Grundgesamtheit für die Stichprobenziehung der Befragung bildeten umfangreiche Callcenter-Datenbanken, die die nationalen Forschungsteams aus den jeweils geeignetsten Quellen erstellten. Der Report kann so keine Repräsentativität beanspruchen, liefert jedoch die bestmögliche Annäherung an ein umfassendes Branchenbild. Die wichtigsten Ergebnisse finden sich in Batt et al. (2009a).

Ursula Holtgrewe, PD Dr., ist Teamleiterin für "Arbeit, Organisation, Internationalisierung " an der Forschungs- und Beratungsstelle Arbeitswelt (FORBA), Wien. Arbeitsschwerpunkte: Dienstleistungsarbeit und -organisation, Internationalisierung von Arbeit, Wissensarbeit, Organisationstheorie, Organisation und Subjektivität, Geschlechterforschung. e-mail: holtgrewe@forba.at Hannelore Mottweiler, Sozialwissenschaftlerin, ist wissenschaftliche Mitarbeiterin am Institut für Soziologie der Universität Duisburg-Essen. Arbeitsschwerpunkte: Arbeitsund Organisationssoziologie, Wirtschaftssoziologie und Vergleichende Soziologie. e-mail: hannelore.mottweiler@uni-due.de Jessica Longen, Sozialwissenschaftlerin, ist Doktorandin am Graduiertenkolleg Topologie der Technik der Technischen Universität Darmstadt. Arbeitsschwerpunkte: Arbeitsund Organisationssoziologie, Techniksoziologie.

e-mail: longen@ifs.tu-darmstadt.de Annika Schönauer, Soziologin, ist wissenschaftliche Mitarbeiterin bei FORBA, Wien. Arbeitsschwerpunkte: Dienstleistungsarbeit und -organisation, Internationalisierung und Flexibilisierung von Arbeit. e-mail: schoenauer@forba.at 


\section{Prognosen und Perspektiven}

Die grenzüberschreitende Restrukturierung von Arbeit und Organisation wird sowohl in Arbeiten zur Globalisierung als auch in Studien zur Relevanz von Regionen und lokalen Faktoren diskutiert. Dennoch werden erst in jüngster Zeit unternehmensübergreifende Untersuchungen zu Outsourcingprozessen in Europa durchgeführt. Auch die Restrukturierung von Dienstleistungen rückt erst neuerdings ins Interesse der Forschung. Hier liegt der Schwerpunkt auf Business Process Outsourcing und Informationstechnik (IT). Diese Forschungsrichtung konzentriert sich überwiegend auf den englischsprachigen Raum (Dossani/Kenney 2003; Taylor/ Bain 2006) und verweist auf die zentrale Rolle Indiens als Outsourcing-Zielland für verschiedenste unternehmensbezogene Dienstleistungen. Nur wenige Untersuchungen befassen sich dagegen mit den Folgen der „globalen“ Arbeitsteilung für die Qualität und Gestaltung der Beschäftigungsverhältnisse und der Arbeit (Marchington et al. 2005; Batt et al. 2009a; Flecker et al. 2008).

Der Schwerpunkt der Forschung über transnationale DienstleistungsRestrukturierungen im englischsprachigen Raum lässt jedoch offen, ob sich die entsprechenden Tendenzen auf andere Regionen übertragen lassen und ob sich Unternehmensstrategien global zu einem anglo-amerikanischen (oder anglo-amerikanisch-indischen) Geschäftsmodell hin entwickeln. Die Annahme einer solchen Konvergenz liegt vielen Globalisierungsprognosen zugrunde. Unter diesem Blickwinkel wäre in Kontinentaleuropa mit einer Intensivierung von Outsourcing zu rechnen.

Die meisten Untersuchungen zur Dienstleistungs-Restrukturierung beobachten dabei eine Spirale von Standardisierung und Outsourcing bzw. Offshoring (Huws et al. 2009). Das muss nicht Dequalifizierung der Arbeit bedeuten: In den Daten des GCC weisen indische Callcenter sowohl hoch standardisierte Arbeit als auch vergleichsweise hoch qualifizierte Agenten auf (Batt et al. 2006). Es bedeutet aber, transnational ebenso wie bei Outsourcing innerhalb eines Landes, prob- lematische Arbeitsbedingungen: Wenig Handlungsspielräume, kleinteilige elektronische Überwachung und niedrige Löhne - also das Gegenteil "guter Jobs“ (Batt et al. 2009b). Aus Sicht der OutsourcingZielländer und der dort Beschäftigten ist dann die Frage, unter welchen Voraussetzungen es möglich wird, die Wertschöpfungskette "hochzuklettern“ und doch eine qualitätsorientierte high road der Dienstleistungs-Restrukturierung einzuschlagen (Dossani/Kenney 2003; Gadrey 2005): anspruchsvollere Aufgaben zu übernehmen, die Arbeitsqualität zu verbessern und outgesourcte Dienstleistungen zu professionalisieren.

\section{3 \\ Die internationale Callcenter-Landschaft}

In den folgenden Abschnitten gehen wir den Fragen nach, in welchem Ausmaß Callcenter international Märkte bedienen, wie sich die Profile nationaler und transnationaler Callcenter unterscheiden und inwiefern der Standort Indien mit gut ausgebildeten Agenten, kleinteilig reglementierter und in hohem Maße standardisierter Arbeit „stilbildend“ für andere Offshoring-Regionen wirkt. Die nachfolgenden Analysen stützen sich auf Daten des GCC (vgl. Fußnote 2). Sie zeigen insgesamt ein begrenztes $\mathrm{Ma}$ an Internationalisierung. In lediglich gut $13 \%$ der Fälle bedient das Callcenter einen internationalen Markt (Tabelle 1).

Markant höhere Anteile lassen sich nur in den englischsprachigen Ländern finden, die als klassische Offshoring-Standorte bekannt sind und vor allem US-amerikanische oder britische Kunden bedienen. Hervorzuheben ist hierbei vor allem Indien mit einem Anteil von $73 \%$ internationaler Callcenter, gefolgt von Irland (37,2 \%) und Kanada (35,1 \%). ${ }^{3}$ Anders als erwartet zählten Länder wie Südafrika und Polen (Zeitpunkt: 2005) nicht zu bevorzugten Offshoring-Regionen. Hier sind die Anteile international arbeitender Callcenter mit 8,6 \% (Süd-Afrika) bzw. 4 \% (Polen) noch relativ gering.

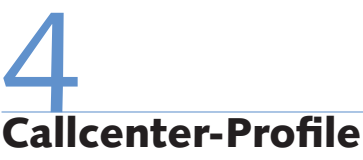

Um unter den Callcentern unterschiedliche Profile zu identifizieren und herauszufinden, wie sich transnational agierende Callcenter von anderen unterscheiden, haben wir eine K-mean-Clusteranalyse

3 Eventuelle Abweichungen der hier dargestellten Daten von den Zahlen in Batt et al. 2009 sind auf Verbesserungen des Datensatzes seit der Veröffentlichung dieser Arbeit zurückzuführen.

\section{Tabelle 1: Nationale und internationale Marktausrichtung von}

Callcentern

\begin{tabular}{|c|c|c|c|c|}
\hline \multirow[t]{2}{*}{ Land } & \multicolumn{2}{|c|}{ Nationaler Markt } & \multicolumn{2}{|c|}{ Internationaler Markt } \\
\hline & $\%^{*}$ & $\mathrm{~N}^{* *}$ & $\% *$ & $\mathrm{~N}^{* *}$ \\
\hline Österreich & 85,4 & 82 & 14,6 & 14 \\
\hline Brasilien & 97,4 & 111 & 2,6 & 3 \\
\hline Kanada & 64,9 & 248 & 35,1 & 134 \\
\hline Dänemark & 96,6 & 112 & 3,4 & 4 \\
\hline Frankreich & 89,5 & 188 & 10,5 & 22 \\
\hline Deutschland & 86,2 & 131 & 13,8 & 21 \\
\hline Indien & 26,7 & 16 & 73,3 & 44 \\
\hline Irland & 62,8 & 27 & 37,2 & 16 \\
\hline Israel & 92,5 & 74 & 7,5 & 6 \\
\hline Japan & 94,8 & 145 & 5,2 & 8 \\
\hline Korea & 99,2 & 120 & 0,8 & 1 \\
\hline Polen & 96,0 & 72 & 4,0 & 3 \\
\hline Südafrika & 91,4 & 53 & 8,6 & 5 \\
\hline Spanien & 84,4 & 92 & 15,6 & 17 \\
\hline Schweden & 90,6 & 126 & 9,4 & 13 \\
\hline Großbritannien & 86,7 & 143 & 13,3 & 22 \\
\hline USA & 98,6 & 434 & 1,4 & 6 \\
\hline Gesamt & 86,8 & 2226 & 13,2 & 339 \\
\hline
\end{tabular}




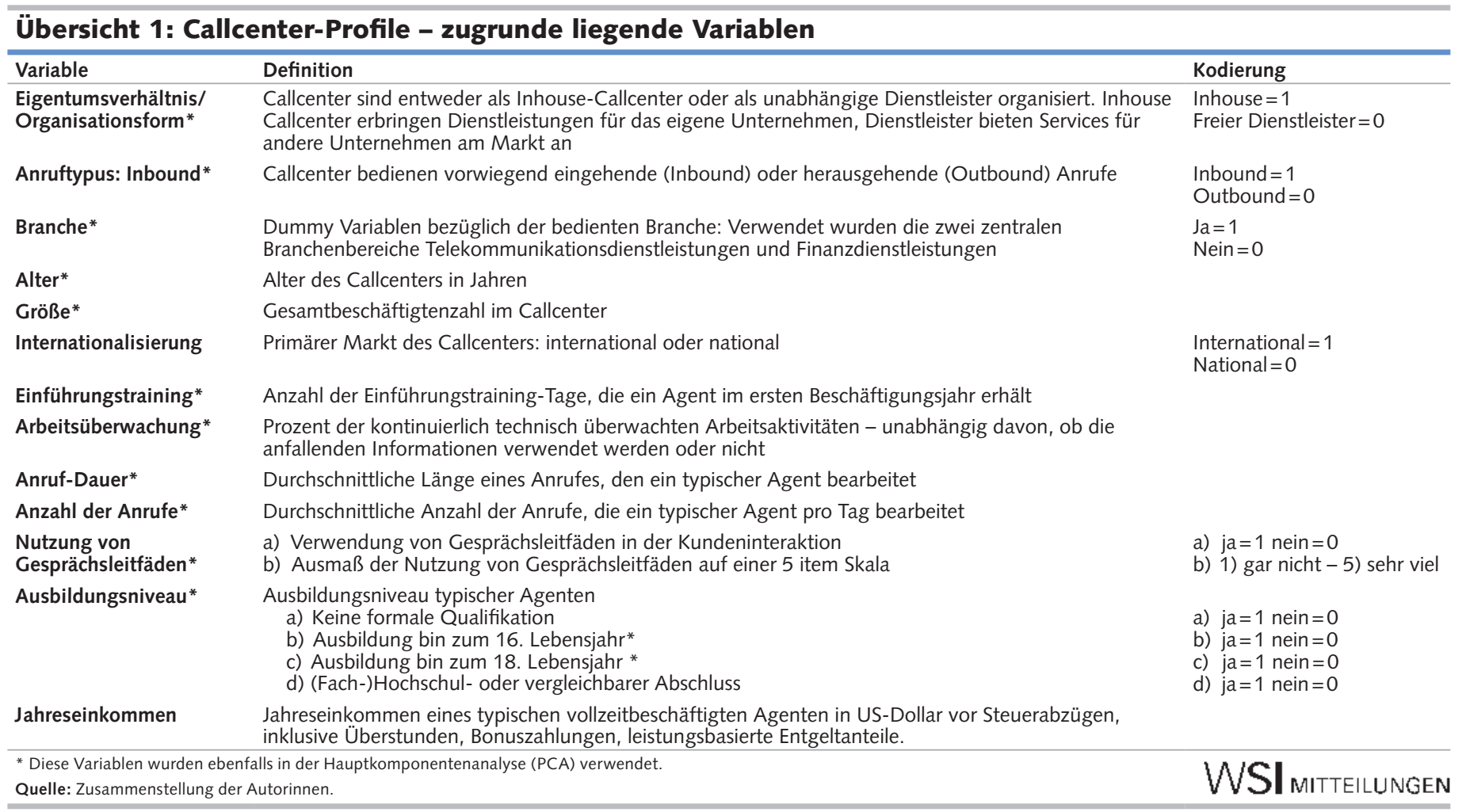

Tabelle 2: Hauptkomponentenanalyse zur Charakterisierung von

Callcenter-Profilen - Rotierte Komponten-Matrix und Faktorenbeschreibung -

\begin{tabular}{|c|c|c|c|c|c|c|}
\hline & & \multicolumn{5}{|c|}{ Komponenten } \\
\hline & & 1 & 2 & 3 & 4 & 5 \\
\hline Alter & Junge, große &,- 479 & & & & \\
\hline Organisationsform (Inhouse) & freie Dienstleister &,- 657 & & & & \\
\hline Arbeitsüberwachung & $\begin{array}{l}\text { mit hoher } \\
\text { Standardisierung und }\end{array}$ &, 412 & & & & \\
\hline $\begin{array}{l}\text { Ausmaß der Nutzung von } \\
\text { Gesprächsleitfäden }\end{array}$ & $\begin{array}{l}\text { hoher Arbeitsüber- } \\
\text { wachung }\end{array}$ & ,649 & & & & \\
\hline Größe & &, 560 & & & ,460 & \\
\hline Anruf-Typus (Inbound) & (Große) Inbound Call- & & & & ,662 & \\
\hline Einführungstraining & $\begin{array}{l}\text { center mit hohen } \\
\text { Investitionen in den } \\
\text { Kompetenzerwerb }\end{array}$ & & & &, 662 & \\
\hline Anruf-Dauer & Geringe Anrufdauer & & & & &,- 733 \\
\hline Anzahl der Anrufe pro Tag & hohes Anrufvolumen & & & & &, 705 \\
\hline Ausbildung bis zum 16. Lebensjahr & (Geringes) & & ,905 & & & \\
\hline Ausbildung bis zum 18. Lebensjahr & Ausbildungsniveau & &,- 912 & & & \\
\hline $\begin{array}{l}\text { Branche: Finanzdienstleistungen } \\
\text { Branche: } \\
\text { Telekommunikationsdienstleistungen }\end{array}$ & $\begin{array}{l}\text { Sektor: } \\
\text { Telekommunikations- } \\
\text { dienstleistungen }\end{array}$ & & & $\begin{array}{r}-, 761 \\
, 799\end{array}$ & & \\
\hline $\begin{array}{l}\text { Eignungsprüfung: Der Barlett-Test zeigte ein } \\
\text { KMO-Wert liegt bei } 0.559 \text {. }\end{array}$ & signifikantes Ergebnis und der & & & & & \\
\hline Quelle: Global Call Center Industry Project; B & erechnungen der Autorinnen. & & & & TTE & UNGEN \\
\hline
\end{tabular}

durchgeführt. Zur Reduzierung der für die Clusteranalyse relevanten Variablenanzahl und zur Eliminierung von Multikollinearität wurde eine Hauptkomponentenanalyse vorgeschaltet. ${ }^{4}$ Einbezogen wurden zentrale Variablen der Organisationsstruktur und der Arbeitsorganisation, die aufschlussreich für die Standardisierung der Arbeit und bestimmte Arbeitsbedingungen der Agenten sind (Übersicht 1; Tabelle 2; Holtgrewe et al. 2009).
Unter Verwendung von orthogonaler rung konnten mithilfe der Hauptkomponentenanalyse fünf Komponenten mit einem Eigenwert größer als 1 extrahiert werden. Diese Komponenten wurden der K-mean-Clusteranalyse als Variablen zugrunde gelegt. Als Ergebnis erhielten wir vier verschiedene Callcenter-Cluster, die im groben mit existierenden CallcenterTypologien übereinstimmen (vgl. etwa Varimax-Rotation mit Kaiser-Normie-
Brasse et al. 2002). Zentrale Unterschiede bestehen zwischen Inhouse-Callcentern und freien Dienstleistern sowie Callcentern mit höherem oder geringerem Qualifikationsniveau (Tabelle 3).

Cluster 1 beinhaltet mittelgroße Callcenter-Dienstleister mit einem hohen Anteil an Outbound-Dienstleistungen, geringen Investitionen in die Qualifizierung der Mitarbeiter, einem hohen Ausmaß an Überwachung und Standardisierung der Arbeit u. a. durch Nutzung standardisierter Gesprächsleitfäden. Die Anzahl an Gesprächen, die ein Agent pro Tag bearbeitet, ist mit durchschnittlich 92 Anrufen geringfügig niedriger als im Gesamtdurchschnitt, der bei 96 bearbeiteten Anrufen pro Tag liegt. Das mittlere Alter

4 Üblicherweise erfordert eine Hauptkomponentenanalyse (PCA) Variablen mit metrischem Skalenniveau. Nach Krzanowski (1984) ist es jedoch auch möglich, ordinal skalierte- und Dummy Variablen in eine PCA zu integrieren, wenngleich es dadurch zu Problemen bei der Dateninterpretation und statistischen Zuverlässigkeit der Ergebnisse kommen kann. In solchen Fällen wäre eine Korrespondenzanalyse eine mögliche Alternative. Da wir im Kontext dieser Datenanalyse die Hauptkomponentenanalyse hauptsächlich zur Verringerung der Variablenzahl und zur Eliminierung von Multikollinearität verwenden und die Typenbildung auf Basis einer K-mean-Clusteranalyse erfolgt, erscheint uns die Verwendung einer Hauptkomponentenanalyse hier angemessen. 


\section{Tabelle 3: Callcenter-Profile nach Clustern}

\begin{tabular}{|c|c|c|c|c|}
\hline Cluster & $\begin{array}{l}\text { junge, standardi- } \\
\text { sierte Dienstleister }\end{array}$ & $\begin{array}{l}\text { große, alte Inhouse- } \\
\text { Telekommunikations- } \\
\text { Callcenter }\end{array}$ & $\begin{array}{l}\text { alte, standardisier- } \\
\text { te, hochvolumige } \\
\text { Callcenter }\end{array}$ & $\begin{array}{l}\text { qualifizierte Inhouse- } \\
\text { Inbound-Callcenter }\end{array}$ \\
\hline Gültige Fälle (Anzahl) & 391 & 422 & 53 & 730 \\
\hline \multicolumn{5}{|l|}{ Standardisierte Mittelwerte der Faktoren } \\
\hline $\begin{array}{l}\text { PCA Faktor } 1 \\
\text { (große, standardisierte, freie DL mit hoher Arbeitsüberwachung) }\end{array}$ & 830 &,- 266 &, 135 &,- 359 \\
\hline $\begin{array}{l}\text { PCA Faktor } 2 \\
\text { (geringes Ausbildungsniveau) }\end{array}$ & ,013 &,- 022 &,- 344 & ,0164 \\
\hline $\begin{array}{l}\text { PCA Faktor } 3 \\
\text { (Telekommunikationsdienstleistungen) }\end{array}$ &,- 166 & 1,151 & 1,051 &,- 669 \\
\hline $\begin{array}{l}\text { PCA Faktor } 4 \\
\text { (Inbound Dienstleistungen mit hohen Qualifizierungs- } \\
\text { investitionen) }\end{array}$ & $-1,063$ & ,364 &,- 592 & ,349 \\
\hline $\begin{array}{l}\text { PCA Faktor } 5 \\
\text { (geringe Anrufdauer, hohes Anrufvolumen) }\end{array}$ &,- 196 &,- 360 & 2,934 &, 116 \\
\hline \multicolumn{5}{|l|}{ Deskriptive Statistiken } \\
\hline Inhouse & $24,4 \%$ & $76,5 \%$ & $52,8 \%$ & $89,0 \%$ \\
\hline Freie Dienstleister & $75,6 \%$ & $23,5 \%$ & $47,2 \%$ & $11,0 \%$ \\
\hline Inbound & $30,2 \%$ & $95,2 \%$ & $83,0 \%$ & $97,0 \%$ \\
\hline Outbound & $69,8 \%$ & $4,8 \%$ & $17,0 \%$ & $3,0 \%$ \\
\hline Telekommunikation & $13,8 \%$ & $91,5 \%$ & $45,3 \%$ &, $0 \%$ \\
\hline Schulausbildung bis $16 \mathrm{~J}$. & $35,8 \%$ & $33,6 \%$ & $35,8 \%$ & $32,3 \%$ \\
\hline Schulausbildung bis $18 \mathrm{~J}$ & $45,5 \%$ & $47,9 \%$ & $58,5 \%$ & $45,5 \%$ \\
\hline College- oder (Fach-) Hochschulabschluss & $17,1 \%$ & $17,8 \%$ & $5,7 \%$ & $20,8 \%$ \\
\hline Alter des Callcenters (Mittelwert) & 7,6 & 14,3 & 17,1 & 10,5 \\
\hline Größe: Anzahl der Mitarbeiter (Mittelwert) & 191,4 & 235,4 & 126,4 & 133,7 \\
\hline Anruf Dauer in Sekunden, (Mittelwert) & 337,8 & 373,1 & 113,1 & 221,6 \\
\hline $\begin{array}{l}\text { Durchschnittliche Anrufanzahl, die ein Agent pro Tag bearbeitet } \\
\text { (Mittelwert) }\end{array}$ & 91,9 & 65,5 & 594,4 & 78,1 \\
\hline Einführungstraining, Tage (Mittelwert) & 9,7 & 17,8 & 11,3 & 16,3 \\
\hline Ausmaß: Verwendung Gesprächsleitfäden (Mittelwert) & 3,51 & 2,26 & 3,04 & 2,06 \\
\hline Arbeitsüberwachung (Mittelwert) & 69,3 & 52,1 & 77,9 & 60,2 \\
\hline Internationalisierung & $24,0 \%$ & $8,6 \%$ & $5,8 \%$ & $11,0 \%$ \\
\hline Durchschnittlicher Jahreslohn, typ. AgentIn, Vollzeit in US-Dollar & $18.566,52$ & $26.176,98$ & $23.075,44$ & $26.212,23$ \\
\hline
\end{tabular}

dieser Unternehmen liegt bei 7,5 Jahren. Der durchschnittliche Verdienst ist mit 18.566,52 US-Dollar signifikant geringer als in anderen Callcentern. Wir nennen Callcenter dieses Clusters ,junge, standardisierte Dienstleister".

Cluster 2 umfasst überwiegend große Inhouse-Callcenter der Telekommunikationsbranche mit einem durchschnittlichen Alter von 14 Jahren. Viele Unternehmen in diesem Cluster sind in den USA beheimatet. ${ }^{5}$ Die Investitionen in die Qualifizierung der beschäftigten Agenten sind überdurchschnittlich hoch, die Arbeitsüberwachung und das Ausmaß der Standardisierung hingegen gering. Ebenso ist das Gesprächsvolumen am Tag mit 65 Anrufen pro Agent niedriger als im Durchschnitt, und Agenten verdienen hier im Mittel 26.176,98 USDollar. Wir bezeichnen diese Callcenter als "große, alte Inhouse-Telekommunikations-Dienstleister".
Im Cluster 3 finden wir sowohl InhouseCallcenter als auch externe Dienstleister. Charakteristisch für diese Gruppierung von Callcentern, die durchschnittlich 17 Jahre alt sind, ist neben der geringen Unternehmensgröße vor allem eine auffallend kurze Gesprächsdauer in Verbindung mit einer äußerst hohen Anzahl an täglich bearbeiteten Anrufen, ein hohes Maß an Gesprächs-Standardisierung und eine überdurchschnittlich hohe Arbeitsüberwachung. Die Agenten erhalten eine vergleichsweise kurze Eingangsschulung mit wenigen Trainingstagen im ersten Jahr. Der Durchschnittsverdienst beträgt hier 23.075,44 US-Dollar. Die Bezeichnung für dieses Cluster lautet: „kleine, standardisierte, hochvolumige Callcenter".

Cluster 4 beinhaltet Inhouse-Callcenter, die Inbound-Dienste im Nicht-Telekommunikationssektor durchführen. Diese Unternehmen mittleren Alters beschäftigen überdurchschnittlich viele (Fach-) Hochschulabsolventen, tätigen hohe In- vestitionen in die Qualifizierung ihrer Mitarbeiter und zeichnen sich durch eine geringe Nutzung von Gesprächsleitfäden und ein geringes Ausmaß an Arbeitsüberwachung aus. Die durchschnittliche Gesprächsdauer in der Kundeninteraktion liegt hier bemerkenswerterweise niedriger als bei den Telekommunikations-Dienstleistern und den ,jungen, standardisierten Dienstleistern", wobei das Anrufvolumen moderat ist. Es ist möglich, dass hier Vorund Nacharbeiten einen Teil der Arbeit ausmachen. Der mittlere Jahresverdienst der Agenten liegt bei 26.212,23 US Dollar. Dieses Cluster nennen wir „qualifizierte Inbound-Inhouse-Callcenter“.

Ausgehend von dieser Typologie untersuchen wir im Folgenden die Beziehungen zwischen Internationalisierung, Eigentumsverhältnissen und Standardisierung

5 Callcenter der Telekommunikationsindustrie sind in der US-amerikanischen Befragung überrepräsentiert (Batt et al. 2009b) 
der Arbeit. Sind es überwiegend die unabhängigen Dienstleister, die internationale Märkte bedienen, weil standardisierte Dienstleistungen sich leichter verlagern und transnational managen lassen? Oder nutzen gerade Callcenter des Typus „qualifizierte Inbound-Inhouse-Center" die Lohnkostenvorteile und Flexibilitätsmöglichkeiten internationalen Agierens? Finden sich hier womöglich länderspezifische Muster der Internationalisierung? Die Frage ist, ob sich in einigen dieser Länder Pfade der Professionalisierung outgesourcter Dienstleistungen auftun (D‘Cruz/Noronha 2006; Dossani/Kenney 2003) oder ob es bei dem Muster hoher Standardisierung und Arbeitskontrolle bleibt. Für den deutschsprachigen Raum mit seinem vorerst geringen Internationalisierungsgrad rechnen wir freilich damit, dass die Internationalisierung sich noch stärker auf die externen Dienstleister beschränkt.

\section{Internationalisierungs- profile}

Die folgenden Analysen untersuchen Internationalisierungsmuster in ausgewählten englisch- und deutschsprachigen Ländern im Vergleich zur Verteilung der Cluster im GCC-Datensatz insgesamt.
Die meisten international operierenden Callcenter sind dem Cluster der ,jungen standardisierten Dienstleister" zuzuordnen (Tabelle 4). Dieser Effekt ist in der Tat in Österreich und in Deutschland stärker als in den englischsprachigen Ländern. Weit überdurchschnittliche vier Fünftel der „internationalen“ Callcenter sind in diesem Cluster angesiedelt. Telekommunikations-Unternehmen und Callcenter des Clusters „kleine, alte hochvolumige Dienstleister" haben hingegen nur einen sehr geringen Internationalisierungsanteil. Im GCC-Datensatz insgesamt sind aber mehr als ein Drittel der international operierenden Callcenter „qualifizierte Inhouse-Inbound-Center". Hier hat Irland ein spezielles Profil: Es hat nahezu zwei Drittel der nationalen wie internationalen Callcenter im „qualifizierten-InhouseInbound"-Cluster und überraschend wenig Unternehmen des Typus ,junge standardisierte Dienstleister". In Indien hingegen ist - wie erwartet - der größte Anteil der Callcenter dem Cluster, ,junge standardisierte Dienstleister" zuzuordnen. Aufgrund insgesamt kleiner Zellenzahlen sind diese Ergebnisse jedoch nur eingeschränkt statistisch robust.

Insgesamt deuten die Daten des GCC nicht auf einen generellen Trend des Offshoring von Callcenter-Dienstleistungen hin. Vielmehr beschränkt sich dieser Trend auf englischsprachige Länder. Insbesondere Irland, Indien und Kanada werden als
Offshoring- oder Nearshoring-Standorte für Callcenter-Dienstleistungen genutzt. Irland profiliert sich als OffshoringStandort für qualifizierte Inhouse-Inbound-Dienste. In anderen Regionen findet Outsourcing hauptsächlich innerhalb desselben Landes statt - aber es ist auch dort mit negativen Folgen für die Arbeitsbedingungen (Holman et al. 2009; Shire et al. 2009) und betriebliche Interessenvertretung (Doellgast et al. 2009; Stern et al. 2010) verbunden. Das irische Profil legt die Schlussfolgerung nahe, dass die qualifizierten „Inhouse-Inbound“-Dienstleistungen auch im englischsprachigen Raum weniger in Niedriglohnregionen als entlang kultureller Ähnlichkeiten verlagert werden. Dementsprechend dürften die Chancen Indiens (und ähnlicher entfernterer Offshoring-Zielländer) vorerst geringer sein, zunehmend qualitätsorientierte Dienste zu übernehmen.

\section{Globaler Kundenservice: Der Fall ABC Electronics}

Zur Illustration der konkreten Standortentscheidungen eines Unternehmens stellen wir im Folgenden das Beispiel der (Inhouse-)Callcenter des multinationalen Konzerns ABC Electronics im deutschsprachigen Raum vor. Es steht für die „qua-

Tabelle 4: Verteilung von nationalen und internationalen Callcentern nach Ländern und Cluster - 2009 -

\begin{tabular}{|c|c|c|c|c|c|c|}
\hline & Cluster & $\begin{array}{l}\text { junge standardisierte } \\
\text { Dienstleister }\end{array}$ & $\begin{array}{c}\text { große, alte Inhouse- } \\
\text { Telekommunikations- } \\
\text { Callcenter }\end{array}$ & $\begin{array}{l}\text { alte, standardisierte, } \\
\text { hochvolumige } \\
\text { Callcenter }\end{array}$ & $\begin{array}{l}\text { qualifizierte Inbound- } \\
\text { Inhouse-Callcenter }\end{array}$ & Anzahl \\
\hline Land & Typus & $\%$ & $\%$ & $\%$ & $\%$ & $\mathrm{~N}$ \\
\hline \multirow[t]{3}{*}{ Österreich } & National & 44,4 & 11,1 & 0,0 & 44,4 & 63 \\
\hline & International & 80,0 & 10,0 & 0,0 & 10,0 & 10 \\
\hline & Alle & 49,3 & 11,0 & 0,0 & 39,7 & 73 \\
\hline \multirow[t]{3}{*}{ Deutschland } & National & 38,2 & 22,1 & 7,4 & 32,4 & 68 \\
\hline & International & 81,8 & 9,1 & 0,0 & 9,1 & 11 \\
\hline & Alle & 44,3 & 20,3 & 6,3 & 29,1 & 77 \\
\hline \multirow[t]{3}{*}{ Kanada } & National & 18,4 & 20,1 & 5,6 & 55,9 & 179 \\
\hline & International & 49,5 & 18,2 & 2,0 & 30,3 & 99 \\
\hline & Alle & 29,9 & 19,2 & 4,3 & 46,6 & 278 \\
\hline \multirow[t]{3}{*}{ Indien } & National & 50,0 & 21,4 & 7,1 & 21,4 & 14 \\
\hline & International & 59,3 & 22,2 & 0,0 & 18,5 & 27 \\
\hline & Alle & 56,1 & 22,0 & 2,4 & 19,5 & 41 \\
\hline \multirow[t]{3}{*}{ Irland } & National & 21,7 & 8,7 & 4,3 & 65,2 & 23 \\
\hline & International & 14,3 & 21,4 & 0,0 & 64,3 & 14 \\
\hline & Alle & 18,9 & 13,5 & 2,7 & 64,9 & 37 \\
\hline \multirow{3}{*}{$\begin{array}{l}\text { GCC insgesamt } \\
\text { (Gültige Fälle) }\end{array}$} & National & 21,4 & 28,1 & 3,6 & 46,9 & 1359 \\
\hline & International & 43,8 & 17,1 & 1,4 & 37,6 & 210 \\
\hline & Alle & 24,4 & 26,6 & 3,3 & 45,6 & 1569 \\
\hline
\end{tabular}


lifizierten Inbound-Inhouse-Callcenter," die im deutschsprachigen Raum eine kleine Minderheit darstellen. Der Fall ist deswegen nicht als „repräsentativ“ für den Cluster anzusehen (was methodisch auch gar nicht möglich ist) - aber er ist aussagekräftig, weil er eine Variante des „,irischen“ Musters regionaler und kultureller Nähe bei den qualifizierten Dienstleistungen für den deutschsprachigen Raum zeigt. 2010 beschäftigt das Unternehmen weltweit 96.000 Mitarbeiter. In den USA ist es nicht nur für seine niedrigen Preise bekannt, sondern auch dafür, dass es sowohl Produktion als auch Service-Center seit Jahrzehnten in Niedriglohnländer verlagert. Zeichnete sich das Unternehmen in der Vergangenheit durch einen Expansionskurs aus, so plante man zum Zeitpunkt der Interviews eine Personaleinsparung von ca. $10 \%$. Neue Kundenservice-Stellen sind meist an den Offshoring-Standorten für den englischsprachigen Markt wie z. B den Philippinen oder Indien entstanden, während in Kanada und den USA Callcenter geschlossen wurden.

Gegen diesen Trend des Stellenabbaus wurden in Europa die Stellen in Callcentern ausgebaut. In Schottland entstand z. B. 2005 ein neuer Standort mit 850 Beschäftigten. Auch in Deutschland und der Slowakei wurde expandiert. Der deutsche Markt von ABC Electronics wird von drei Standorten aus bedient: Aus Ostdeutschland leistet das Unternehmen den Support für Geschäftskunden, im slowakischen Callcenter werden die Anrufe deutscher Privatkunden entgegengenommen, und am Standort in Westdeutschland befindet sich der Vertrieb. Die technische Infrastruktur der Standorte würde es möglich machen, bei Belastungsspitzen Gespräche an den jeweiligen anderen Standort zu leiten. Tatsächlich wird die Segmentierung von Privatkunden und Geschäftskunden jedoch streng aufrechterhalten. Außerhalb der Arbeitszeiten der deutschen Hotline werden Geschäftskunden-Anrufe ins englischsprachige Ausland weitergeleitet. Die Entscheidung des Unternehmens für die beiden Callcenter-Standorte wird mit den niedrigen Miet- und Personalkosten, der technischen Infrastruktur und den Qualifikationen der Beschäftigten, insbesondere den guten deutschen Sprachkenntnissen am slowakischen Standort, begründet.

Der Standort in Ostdeutschland ist der jüngste; er wurde 2005 gegründet, die Ausbauarbeiten dauern an. Zum Zeitpunkt des Interviews arbeiteten fast 700 Mitarbeiter dort, davon 270 im Support. Bei der Beschäftigtenstruktur zeigt sich das typische Muster für technische Hotlines, nämlich ein hoher Anteil männlicher Beschäftigter in Vollzeitbeschäftigung mit abgeschlossenen Berufsausbildungen. Der Anteil studentischer Arbeitskräfte ist gering. Diese Mitarbeiterstruktur erklärt sich durch die hohe Arbeitslosenquote in der Region $(15,2 \%$ vs. $8,1 \%$ in Gesamtdeutschland zum Zeitpunkt der Interviews) und durch ein entsprechend niedriges Lohnniveau. Die Arbeit selbst unterscheidet sich von „typischen" Callcentern und auch von anderen IT-Hotlines. Beschäftigte, die gerade nicht telefonieren, diskutieren mit anderen technische Probleme. Obwohl die Arbeit sehr fragmentiert ist, sind die Gespräche wenig standardisiert, und auch der Zeitdruck ist sehr niedrig. Gleichwohl werden Gespräche und Eingaben der Agenten am Computer überwacht. Das erklärt sich durch die Spezialisierung des Standortes auf Geschäftskunden und auch durch die Besonderheit des Aufgabengebietes. Die Strategie, Geschäfts- und Privatkunden durch unterschiedliche Center bedienen zu lassen, verfolgt das Unternehmen weltweit.

Der slowakische Standort, an dem die Privatkunden bedient werden, ist deutlich größer. Zum Zeitpunkt der Interviews arbeiteten dort insgesamt ca. 2000 Beschäftigte, nicht alle als Callcenter-Agenten. Er wurde 1997 als reines Service-Center in einer slowakischen Stadt gegründet, die sich inzwischen als Nearshoring-Standort für deutschsprachige Callcenter-Dienste etabliert hat. Bei der Umwandlung des Standortes 2002 in ein Business Center für die Gebiete Europa, Naher Osten und Afrika wurden 800 neue Stellen geschaffen. Seit 2003 wird der deutschsprachige Kundenservice für Privatkunden ausschließlich dort angeboten.

Bei ABC Electronics mit seinen vergleichsweise qualifizierten SupportDienstleistungen ist also die Verteilung der Standorte nicht mit einer reinen Kostensenkungsstrategie zu erklären. Kostenorientierung bei der Standortwahl und differenzierte Reaktion auf unterschiedliche Märkte treffen aufeinander. Die Expansion des Unternehmens auf den nicht-englischsprachigen Markt geht mit einem Ausbau des entsprechenden Kundenservices einher, während man im englischsprachigen Kundenservice Standorte zusammenlegt. Daneben zeigt das Beispiel, dass ein niedriges Lohniveau durchaus mit Handlungs- spielräumen der Beschäftigten in Bezug auf den Arbeitsvollzug und die Anforderungen des IT-Supports für Geschäftskunden einhergehen kann. Die Standortwahl hat dabei vermutlich auch ein Stück weit symbolischen Charakter: Indem Geschäftskunden durch Muttersprachler bedient werden, versucht das Unternehmen deren Bindung an das Unternehmen zu erhöhen und nimmt höhere Lohnkosten in Kauf. Dagegen wird von Privatkunden, obgleich sie im Umgang mit IT-Hotlines in der Regel weit weniger routiniert sind, erwartet, dass sie in Kooperation mit den slowakischen Beschäftigten kulturelle Unterschiede und Verständigungsprobleme überbrücken.

\section{1 \\ Schlussfolgerungen}

Insgesamt ist festzuhalten, dass es zwar transnational agierende Konzerne und Callcenter-Dienstleister gibt, aber dass diese nur einen vergleichsweise kleinen Teil des Gesamtbildes prägen. Überwiegend sind es große, junge Callcenter-Dienstleister, die auf einem transnationalen Markt tätig sind und sich im eher standardisierten Teil des Callcenter-Dienstleistungsspektrums bewegen. Bei qualifizierten Dienstleistungen, die innerhalb eines Unternehmens erbracht werden, werden Kostensenkungslogiken durch kulturelle Nähe gedämpft. Transnationalisierung beschränkt sich hier auf bestimmte Dienste, die von ihrer Beschaffenheit her schon transnationaler sind, wie Flugbuchungen oder eben IT-Hotlines. Selbst im englischsprachigen Raum geht eine Vielzahl von Standortverlagerungen eher in die Nachbarschaft, von den USA nach Kanada oder von Großbritannien nach Irland - aber dorthin werden auch qualifiziertere Dienstleistungen verlagert. Das bedeutet allerdings auch, dass entferntere Offshoring-Regionen nur schwache Aussichten haben, qualifiziertere Arbeiten $\mathrm{zu}$ übernehmen und Callcenter mit „besseren Jobs“ anzuziehen. Es sei denn, die transnationale Dienstleister-Branche schlüge im Bündnis mit anspruchsvollen Kunden einen dezidierten Qualitätspfad ein, was uns unwahrscheinlich erscheint.

Für den deutschsprachigen Raum wissen wir bislang noch wenig über Strategien der Standortverlagerung einerseits, des Nearshoring an ost- oder südosteuropäische Dienstleister andererseits. Dazu 
bräuchte es detailliertere Daten über Eigentümerbeziehungen, Vernetzungen, internen und externen Wettbewerb zwischen Callcentern. Dass aber die Transnationalisierung weitergehen wird, ist absehbar. Prognosen von VerlagerungsExplosionen nach Ost- und Südosteuropa dürften jedoch übertrieben sein, wiewohl die Branche dort durchaus noch wächst. Einstweilen sind es Telekommunikationsunternehmen, technische Hotlines und Callcenter-Dienstleister, die in den neuen
EU-Mitgliedsländern Callcenter eröffnen (Holtgrewe 2009). Insgesamt rechnen wir mit moderaten länderübergreifenden Verlagerungen in folgenden Bereichen: Bei den jungen, womöglich auch den älteren standardisierten Dienstleistern und ihren Auftraggebern, also den einfachen, geringer entlohnten und hochvolumigen Diensten. In zweiter Linie bei bestimmten, durchaus auch qualifizierten Inbound-Diensten wie IT oder Reise. Möglich ist auch, dass in Süd- und Osteuropa, wo transnationale
Telekommunikations-, Handels- oder Finanz-Dienstleister zuerst nationale Märkte erschlossen haben, diese nun ihre Callcenter-Operationen an Standorten mit niedrigen Kosten konsolidieren. Sowohl zukünftige Untersuchungen als auch die gewerkschaftliche Praxis werden demnach auf mittlere Sicht gut daran tun, die Möglichkeiten und Wirklichkeiten in den Blick zu nehmen, die mit der Restrukturierung von Arbeit über betriebliche und regionale Grenzen hinweg verbunden sind.

\section{LITERATUR}

Batt, R./Doellgast, V./Kwon, H. (2006): Service Management and Employment Systems in U.S. and Indian Call Centers, in: Collins, S. M./ Brainard, L. (Hrsg.): Brookings Trade Forum 2005: Offshoring White-Collar Work - The Issues and Implications, Washington D.C., S. 335-372

Batt, R./Holman, D./Holtgrewe, U. (Hrsg.) (2009a): The Globalization of Service Work: Comparative Institutional Perspectives on Call Centers, Industrial \& Labor Relations Review 62 (4), special issue

Batt, R./Holman, D./Holtgrewe, U. (2009b): The Globalization of Service Work: Comparative Institutional Perspectives on Call Centers. Introduction to the ILRR special issue, in: Industrial \& Labor Relations Review 62 (4), S. 453-488

Brasse, C./Engelbach, W./Schietinger, M./Schmitz, E. (2002): AKLTypologie. Ein empirischer Ansatz zur Typologisierung von Call Centern, Dortmund, http://www.incca.iao.fhg.de/Ergebnisse/Typologie.pdf. D‘Cruz, P./Noronha, E. (2006): Being Professional: Organizational Control in Indian Call Centers, in: Social Science Computer Review 24 (3), S. 342-361

Djelic, M.-L./Quack, S. (Hrsg.) (2003): Globalization and Institutions. Redefining the Rules of the Economic Game, Cheltenham UK/Northampton MA

Doellgast, V./Holtgrewe, U./Deery, S. J. (2009): The effects of national institutions and collective bargaining arrangements on job quality in frontline service workplaces, in: Industrial \& Labor Relations Review 62 (4), S. 489-509

Dossani, R./Kenney, M. (2003): Went for Cost, Stayed for Quality? Moving the Back Office to India, Stanford CA, http://iis-db.stanford. edu/pubs/20337/dossani_kenney_09_2003.pdf

Flecker, J./Holtgrewe, U./Schönauer, A./Dunkel, W./Meil, P. (2008): Restructuring across value chains and changes in work and employment. Case study evidence from the Clothing, Food, IT and Public Sector, WORKS WP 10, Deliverable 10.1., Leuven, http://www. worksproject.be/Subgroup_3_proj_reports.htm.

Gadrey, J. (2005): Diversity and regulation of markets for services, in: Bosch, G./Lehndorff, S. (Hrsg.): Working in the Service Sector. A Tale from Different Worlds, Abingdon, S. 342-353
Holman, D./Frenkel, S. J./Sørensen, O. H. (2009): Work design variation and outcome in call centres: Strategic choice and institutional explanations, in: Industrial \& Labor Relations Review 62 (4), S. 510-532 Holtgrewe, U. (2009): Callcenter: nicht ganz global, aber hochflexibel. Ein internationaler Überblick, in: Ahlers, E./Kraemer, B./Ziegler, A. (Hrsg.): Beschäftigte in der Globalisierungsfalle?, Wiesbaden, S. $137-155$

Holtgrewe, U./Longen, J./Mottweiler, H./Schönauer, A. (2009): Global or embedded service work: the (limited) transnationalisation of the call-centre industry, in: Work, Organisation, Labour and Globalisation 3 (1), S. 9-25

Huws, U./Dahlmann, S./Flecker, J./Holtgrewe, U./Schönauer, A./ Ramioul, M./Geurts, K. (2009): Value chain restructuring in Europe in a global economy, Leuven, http://worksproject.be/Works_pdf/ WP12 \%20publiek/09_D12.1\%20Thematic\%20Report_Global ValueChains_DRUK.pdf

Krzanowski, W. J. (1984): Principal Components Analysis in the Presence of Group Structure, in: Applied Statistics 33 (2), S. 164-168 Marchington, M./Grimshaw, D./Rubery, J./Willmott, H. (Hrsg.) (2005): Fragmenting Work. Blurring Organizational Boundaries and Disordering Hierarchies, Oxford

Shire, K. A./Schönauer, A./Valverde, M./Mottweiler, H. (2009): Collective Bargaining and Temporary Contracts in Call Centre Employment in Austria, Germany and Spain, in: European Journal of Industrial Relations 15 (4), S. 437-456

Sørensen, O. H./Weinkopf, C. (2009): Pay and Working Conditions in Finance and Utility Call Centres in Denmark and Germany, in: European Journal of Industrial Relations 15 (4), S. 395-416

Stern, S./Schönauer, A./Holtgrewe, U. (Hrsg.) (2010): Service um jeden Preis? Arbeiten im Callcenter. Erfahrungsberichte und Organisationsmöglichkeiten, Wien

Taylor, P./Bain, P. (2006): Work organisation and employee relations in Indian call centres, in: Burgess, J./Connell, J. (Hrsg.): Development in the call centre industry: analysis, changes and challenges, New York/ London, S. 36-57 